\title{
Quantum interference in graphene
}

\section{nanoconstrictions}

Pascal Gehring ${ }^{1, *}$, Hatef Sadeghi ${ }^{2}$, Sara Sangtarash ${ }^{2}$, Chit Siong Lau ${ }^{1}$, Junjie Liu ${ }^{1}$, Arzhang Ardavan ${ }^{3}$, Jamie H. Warner ${ }^{1}$, Colin J. Lambert ${ }^{2}$, G. Andrew. D. Briggs ${ }^{1}$, Jan A. Mol ${ }^{1}$

${ }^{1}$ Department of Materials, University of Oxford, 16 Parks Road, Oxford OX1 3PH, United Kindom

${ }^{2}$ Quantum Technology Centre, Physics Department, Lancaster University, Lancaster LA1 4YB, United

Kingdom

${ }^{3}$ Clarendon Laboratory, Department of Physics, University of Oxford, Parks Road, Oxford OX1 3PU, United Kingdom

pascal.gehring@materials.ox.ac.uk

\begin{abstract}
We report quantum interference effects in the electrical conductance of chemical vapour deposited graphene nanoconstrictions fabricated using feedback controlled electroburning. The observed multi-mode Fabry-Pérot interferences can be attributed to reflections on potential steps inside the channel. Sharp anti-resonance features with a Fano line shape are observed. Theoretical modelling reveals that these Fano resonances are due to localised states inside the constriction, which couple to the delocalised states that also give rise to the Fabry-Pérot interference patterns. This study provides new insight into the interplay between two fundamental forms of quantum interference in graphene nanoconstrictions.
\end{abstract}

KEYWORDS: graphene, quantum interference, Fano resonance, break junction, Fabry-Pérot

A key feature of electron transport through single molecules and phase-coherent nanostructures is the appearance of transport resonances associated with quantum interference.[1] Examples include BreitWigner resonances, multi-path Fabry-Pérot resonances and Fano resonances. Fano resonances can be observed when a localised state interacts with a continuum of extended states and can lead to very steep gradients in the transmission. Unlike Breit-Wigner resonances, they are not life-time broadened 
by coupling to the electrodes. The steep slope of Fano resonances makes them attractive for lowpower switching and for creating structures with high thermoelectric performance.[2] In what follows, we report the first observation of Fano resonances in electroburnt graphene nanoconstrictions. In addition to these Fano features, the conductance maps exhibit interference patterns which we attribute to multi-mode Fabry-Pérot (FP) interferences. Theoretical modelling reveals that the Fano resonances arise from interaction between the delocalised state giving rise to the Fabry-Pérot pattern and a localised state inside the constriction.

Carbon-based nanostructures, such as metallic or semiconducting single carbon chains[3,4], graphene nanoribbons and graphene nanoconstrictions are interesting platforms for the study of spintronics[5] and might enable novel technological applications[6]. Graphene nanoconstrictions and nanogaps also provide a robust platform for studying the electric[7], thermoelectric[8] and magnetic[9] properties of single molecules. When sufficiently narrow, graphene nanoribbons can be used to build field-effect transistors with an on/off ratio that can exceed 1000.[10] In very narrow constrictions, with a width smaller than the electronic wavelength of electrons, quantum interference effects in analogy to subwavelength optics are predicted[11,12]. Graphene nanoconstrictions have been fabricated by means of electron beam lithography[13], gold break-junction etching masks[10], local gating[14] and electroburning of graphene[15,16]. Electroburning has also been used to fabricate graphene quantum dots with addition energies up to $1.6 \mathrm{eV}$, enabling the observation of Coulomb blockade at room temperature[17]. In this study we use feedback-controlled electroburning to narrow down lithographically-defined bowtie shaped graphene constrictions[18] and study their electronic transport behaviour.

Our devices are fabricated from single-layer CVD-grown graphene[19] which we transfer onto a $\mathrm{Si} / 300 \mathrm{~nm} \mathrm{SiO}{ }_{2}$ wafer with pre-patterned $10 \mathrm{~nm} \mathrm{Cr} / 70 \mathrm{~nm} \mathrm{Au}$ contacts. We pattern the graphene into a bowtie shape (see Figure 1a,b) using standard electron beam lithography and $\mathrm{O}_{2}$ plasma etching. The channel length $L$ of the devices and the width $W$ of the narrowest part of the constriction are $4 \mu \mathrm{m}$ and $200 \mathrm{~nm}$, respectively (see Figure 1a). Our devices are p-doped with a Dirac point $V_{\text {Dirac }}$ around 60 V (see Figure 1c). The single-layer nature of the graphene constriction is confirmed by the intensity 
ratio $I(2 \mathrm{D}) / I(\mathrm{G}) » 1$ of the Raman $\mathrm{G}$ and $2 \mathrm{D}$ peaks (see Figure $1 \mathrm{~d}$ ) and the fact that the $2 \mathrm{D}$ peak consists of a single Lorentzian.[20,21] In addition, we observe a D and D' peak which we attribute to the defective graphene edges formed during the plasma etching.[21] These defect peaks are not present in bulk single-layer graphene samples.[19] To narrow down the constriction we use a feedback-controlled electroburning technique in air, similar to the one described in Ref [18]. We ramp-up a voltage applied between the source and drain contact while monitoring the current with a 5 $\mathrm{kHz}$ sampling rate (see Figure 1b). As soon as a drop in the current is detected, the voltage is quickly ramped back to zero. This cycle is repeated until the low bias source-drain resistance of the device, which is measured after each burning cycle, exceeds a threshold resistance of $500 \mathrm{M} \Omega$. The feedback conditions are adjusted for each burning cycle depending on the threshold voltage $V_{\text {th }}$ at which the drop in the previous cycle occurred. The current-voltage $\left(I-V_{\mathrm{b}}\right)$ traces of a typical electroburning process are shown in Figure 1e, where the $I-V_{\mathrm{b}}$ traces before electroburning and after the threshold resistance is reached are coloured blue and red, respectively.

During electroburning, the constriction is narrowed down and as a result the resistance of the device increases. At the final stage, the (only several atoms wide) constriction can break completely and a nanometre sized gap is formed.[12] However, for many devices the threshold resistance is reached before a gap is fully formed. In these cases, narrow graphene constrictions or small graphene islands are left between the mesoscopic graphene leads. Graphene quantum dots formed in this manner have been widely studied[15-17,22] as a possible platform for room temperature single-electron transistors. In the following we discuss the details of the transport characteristics of empty graphene nanogaps, quantum dots and nanoconstrictions recorded at $T=4 \mathrm{~K}$ in vacuum $\left(\sim 10^{-6} \mathrm{mbar}\right)$.

The transport regime which we attribute to an empty gap is characterised by low currents and $I-V_{\mathrm{b}}$ characteristics that can be fitted using the standard Simmons model[23] for tunnelling through a single trapezoidal barrier between source and drain (see Figure 2a). In addition, the $I-V_{\mathrm{b}}$ characteristics show no or a relatively small back gate dependence (see Figure $2 b$ ). We find gap sizes of $0.5-2.5 \mathrm{~nm}$ for these junctions, making them a promising platform for single molecule electronics.[7,24,25] 
Devices in the weakly coupled quantum dot regime show suppressed current at low bias (see Figure 2c) and characteristic Coulomb diamonds as a function of bias and gate voltage (see Figure $2 \mathrm{~d}$ ). These transport features are indicative of sequential electron tunnelling via a weakly coupled quantum dot between source and drain.[26] From the size of the Coulomb diamonds we can extract addition energies for these quantum dots ranging from 20 to $800 \mathrm{meV}$, comparable to those found by other groups in similar systems.[13,15-17] The formation of graphene quantum dots during electroburning process is the result of electron/hole localisation due to charge puddles and/or edge disorder as the graphene channel gets narrower.[27] Theoretical calculations have also shown that localised states can form along the edges of wedge-shaped nanoconstrictions.[28] Furthermore, it is possible that small graphene islands on the order of several $\mathrm{nm}$ form, which are only weakly coupled to the graphene leads.[17]

The conductance maps of strongly-coupled devices are dominated by "chess board"-like interference patterns as shown in Figure 2f. In some samples we could observe a transition from this chess board pattern to a Coulomb diamond regime at high positive gate voltages of $\gtrsim 40 \mathrm{~V}$. This observation is similar to results found in recent studies on short graphene junctions[29,30] and narrow graphene constrictions[31]. In the latter, the chess board pattern was attributed to interference effects of extended states in the source or drain graphene lead connecting the constriction.[31] In general, interference effects occur on a length scale on the order of the phase coherence length, but can have different origins. If the transport in the graphene sample is diffusive, i.e. when charge carriers are predominantly scattered at random impurities like edge disorder, point defects or charge puddles,[27] the origin of the interference pattern is most likely due to quantum interferences of different random scattering paths (universal conductance fluctuations, UCFs). If the channel length is on the order of or shorter than the mean free path of the carriers (quasi-ballistic transport regime), reflections in the channel result in quasi-periodic multi-mode or collective and periodic single-mode Fabry-Pérot interferences. Carriers can get reflected at the metal contacts[30] or at potential barriers formed by intentional local doping.[32] Whether single- or multi-mode interference is observed strongly depends on the detailed device geometry.[33]

4 
Fabry-Pérot interference effects have previously been observed in 1D nanowires[34], carbon nanotubes[35] and 2D graphene[30], while UCFs have been observed in mesoscopic single-[36], biand tri-layer[37] and epitaxial graphene samples[38]. To distinguish between these different types of quantum interference, the chess board conductance patterns need to be carefully analysed for hidden periodicities.[30] From the characteristic energy spacing between single features in the conductance maps and Fast Fourier Transforms (FFTs) of the data shown in Figure 2f (see Figure S6a and $b$ in the Supplementary Information) we can extract a typical energy spacing of $4-5 \mathrm{meV}$. Using a particlein-a-box approximation[30] we estimate the relevant length scale $L=h v_{\mathrm{F}} /(2 E)$ to be between $400 \mathrm{~nm}$ for the theoretical local density approximation limit of the Fermi velocity of $v_{\mathrm{F}}=0.8 \times 10^{6} \mathrm{~m} / \mathrm{s}$ and 1.1 $\mu \mathrm{m}$ for a Fermi velocity of $v_{\mathrm{F}}=2.4 \times 10^{6} \mathrm{~m} / \mathrm{s}$ measured for CVD graphene on a quartz substrate.[39] This length scale corresponds to half the minimal distance over which the electrons remain phase coherent, therefore we can infer a lower bound for the phase coherence length $L_{\varphi}>800 \mathrm{~nm}$ in our samples.[29] This value is similar to the value found for exfoliated graphene on $\mathrm{SiO}_{2}$ [40], epitaxial graphene[41] and CVD graphene[42]. For short and wide devices small incident angles dominate (longitudinal modes) and resonances appear at $k_{\mathrm{F}} L=n \pi$.[32] However, since our devices are not in the limit $W / L » 1$, both longitudinal and transversal modes need to be considered. To model conductance maps for different aspect ratios we have performed nearest-neighbour tight-binding calculations[33] (see section S5 Supplementary Information). Our calculations confirm that for $W » L$ a periodic interference pattern with high contrast can be observed. This is due to the fact that the energy of transversal modes $E_{\mathrm{W}}=h v_{\mathrm{F}} /(2 W)$ gets negligibly small. The same holds for the 1D limit $W \rightarrow 0$, where $E_{\mathrm{W}}$ goes to infinity. In both cases the transport is dominated by longitudinal modes only. In the intermediate multi-mode regime, periodic longitudinal modes can still be observed in the FFT but with much smaller contrast. Since the aspect ratio $W / L$ of our devices is close to unity we expect that the interference pattern shown in Figure $2 b$ will only be quasi-periodic because of multi-mode interferences. Moreover, the fact that the width $W$ of the samples is not constant will cause the transversal modes to become chaotic.[11] 
Because the measured chess board pattern is only quasi-periodic, we cannot exclude UCFs as an origin of the observed pattern. UCFs are normally most pronounced at low doping concentrations when the electrochemical potential of graphene is close to the Dirac point.[30] This is unlikely to be the case in our $p$-doped graphene junctions. In addition, the periodicity which we can correlate with the geometry of the device is very similar for all devices investigated in this study, which makes multi-mode Fabry-Pérot interferences a more likely mechanism to explain our data.

Next, we will investigate the microscopic origin of the FP reflections. Based on our assumption for the Fermi velocity (see above) we estimate that carriers are coherently reflected on a length scale of $\square$ $1 \mu \mathrm{m}$. The visibility/intensity of FP interferences is determined by the reflectance of the potential steps. Unipolar cavities have a small finesse and result in a small visibility $\left(G_{\max }-G_{\min }\right) /\left(G_{\max }+\right.$ $\left.G_{\min }\right)$ since the conservation of pseudospin suppresses backscattering in graphene.[32] A smooth bipolar potential step like a $p n$ junction formed near a metal-graphene contact has a much higher finesse and leads to pronounced resonance pattern.[32] However, since the length scale of less than 1 $\mu \mathrm{m}$ found above is much smaller than the channel length of $4 \mu \mathrm{m}$ of our devices there need to be additional potential steps inside the graphene channel apart from the metal contacts. From scanning electron microscopy and micro Raman spectroscopy (see Sections S1 and S4 in the Supplementary Information) we can infer that the local hole concentration within a region of several hundreds of $\mathrm{nm}$ around the graphene constriction is increased during electroburning. The increase of hole doping of graphene on $\mathrm{SiO}_{2}$ annealed in air was intensively studied and attributed to doping by $\mathrm{O}_{2}$ and moisture and a change in the degree of coupling between graphene and $\mathrm{SiO}_{2}$.[43] This increased $\mathrm{p}$ doping can result in the formation of a $p p^{+} p$ junction in the central region of the devices (see Figure S6c and $\mathrm{d}$ in the Supplementary Information). Possible resonance conditions are reflections between the gold contact/the $p n$ junction close to the gold contact and the $p p^{+} p$ junction or reflections within the $p p^{+} p$ junction which all have a characteristic length scale of several hundreds of nm. This length scale is on the order of the-mean-free path of charge carriers in our devices (see Supplementary Information), which further corroborates our interpretation that the chess board pattern arises from FP interferences rather than scattering at random impurities inside the channel. The visibility of the FP interferences 
$\left(G_{\max }-G_{\min }\right) /\left(G_{\max }+G_{\min }\right)>10 \%$ is high in our devices, which indicates that the unipolar $p^{+} p$ interfaces need to have a sharp potential drop with $\mathrm{k}_{\mathrm{F}} d<<1$, where $d$ is the length over which the carrier density changes.[44] We estimate this length scale by calculating the Fermi vector using $n=$ $\mathrm{k}_{\mathrm{F}}{ }^{2} / \pi$ and the charge carrier concentration $n=C_{\mathrm{g}}^{2}\left(V_{\mathrm{g}}-V_{\text {Dirac }}\right)^{2} / e^{2},[30]$ where $C_{\mathrm{g}}$ is the capacitance of the back gate and $e$ is the elementary charge. For $V_{\text {Dirac }}=60 \mathrm{~V}$ (see Figure $1 \mathrm{c}$ ), $d$ is on the order of $3 \mathrm{~nm}$.

We only see interference patterns in nearly fully-burned devices and not directly after the first electroburning steps. We attribute this to the decreasing conductance of the graphene constriction during electroburning, which decreases the denominator in $\left(G_{\max }-G_{\min }\right) /\left(G_{\max }+G_{\min }\right)$ and thus increases the visibility of the interferences. Another possible explanations for the onset of interference pattern after electroburning is the recrystallisation of the constriction,[45] which may lead to a higher mean free path that is required for reflections on the $\mu \mathrm{m}$ scale. The interplay between reduced width and reduced carrier density may also increase the factor $\lambda / W$, where $\lambda=h v_{\mathrm{F}} / E$ is the wavelength of the electrons. If this ratio becomes $\gtrsim 3-5$ the Fabry-Pérot interferences have a high contrast.[11]

We now turn to the sharp anti-resonances in the interference regime as shown in Figure $3 \mathrm{a}$ and $\mathrm{b}$ (around $V_{\mathrm{g}}=-18 \mathrm{~V}$ ) in some samples (see Supplementary Information for data of other samples). The slope of this anti-resonance feature is different from the slopes of the multi-mode FP interference patterns. Repeated thermal cycling from $4 \mathrm{~K}$ to room temperature did not change the slope and position of the feature observed at $4 \mathrm{~K}$ (see Figure S8). The feature consists of an antiresonance/resonance double-peak as shown in Figure 3c. This asymmetric curve has a distinct Fano line shape,[46] which is the result of coherent interaction between a localised resonant state with a delocalised background state.[1] Fano resonances have previously been observed in double donor systems in nanoscale silicon transistors[47] and in bundles of single walled CNTs[48]. Fano resonances are also predicted for single molecule systems, where a backbone state is coupled to the leads and a pendant side-group is only coupled to the backbone but not to the leads.[1] In a graphene constriction connected to mesoscopic graphene leads there are delocalised states that give rise to the previously discussed FP pattern, and bound states e.g. localised along the edges due to edge 
roughness, that give rise to Coulomb blockade at high positive gate voltages close to the Dirac point (see Figure 2f).[31] We attribute the observed Fano resonances to the coherent interaction between these states.

To estimate the coherent coupling strength between the localised and delocalised states in the graphene nanoconstriction, we fit the low bias current - gate voltage $\left(I-V_{g}\right)$ traces to the Fano formula:[48,49]

$$
G(\varepsilon)=G_{\text {non }}+G_{\text {res }} \frac{(\varepsilon+q)^{2}}{\varepsilon^{2}+1}
$$

where $G_{\text {res }}$ is the coherent contribution to the conductance, $q$ is the complex Fano factor,[50] $\varepsilon=$ $2\left(E-\varepsilon_{\mathrm{S}}\right) / \Gamma_{\mathrm{Fano}}, \varepsilon_{\mathrm{S}}$ and $\Gamma_{\mathrm{Fano}}$ are the energy and coupling strength of the resonant localised state and $G_{\text {non }}$ is the conductance of the non-resonant channel. We model the non-resonant background as the sum of a constant offset $G_{\text {off }}$ and a Breit-Wigner peak $A \frac{\Gamma^{2}}{\Gamma^{2}+\left(E-\varepsilon_{\mathrm{b}}\right)^{2}}$. This non-resonant background accounts for the conductance peak close to the observed anti-resonance feature. Fits to our data at different bias voltages using Equation (1) are shown as solid lines in Figure 3c. We find for a low bias of $V_{\mathrm{b}}=0.1 \mathrm{mV}: \varepsilon_{\mathrm{s}}=-18.3 \mathrm{meV}, \operatorname{Re}(q)=0.3, \operatorname{Im}(q)=1.1,|q|=1.1 \Gamma_{\text {Fano }}=0.4 \mathrm{meV}$, and a BreitWigner peak at $\varepsilon_{\mathrm{b}}=-20.5 \mathrm{meV}$ with a coupling strength of $\Gamma=1.1 \mathrm{meV}$ using a lever arm $\mathrm{d} E / \mathrm{d} V_{\mathrm{g}}$ of $1 \mathrm{meV} / \mathrm{V}$ extracted from the slope of the Fabry-Pérot interference pattern as depicted by the dotted black line in Figure 3a. The Fano factor $q$ is a combined measure for the energetic detuning and the ratio of the transmission amplitudes of the resonant and the non-resonant channel.[49] For $q \rightarrow \infty$, the transport is dominated by the resonant channel and the line shape becomes that of a Breit-Wigner peak. For $q \rightarrow 0$ non-resonant transport dominates resulting in a symmetric dip in the conductance.[49] The value of $|q|=1.1$ found in our experiments results in an asymmetric feature with characteristic Fano line-shape.[48] The width of $\Gamma_{\text {Fano }}=0.4 \mathrm{meV}$ of the resonant state is similar to the values of $0.25-0.5 \mathrm{meV}$ found for carbon nanotube bundles.[48] The Fano factor $q$ decreases with increasing positive bias voltage (see inset in Figure 3c) which we attribute to a detuning of the energies of the localised state and the extended states. For large negative bias voltages the detuning 
changes the Fano factor from 1 to a high value, and the transport is dominated by a resonant channel resulting in a Breit-Wigner peak.

The slope of the Fano feature, as seen in Figure 3a, results from the electrostatic coupling of the localised 'pendant' state to the gate and lead electrodes. Figure 4a, shows a tight-binding model of a pendant state interacting with an extended 'backbone' state. A chain of 5 sites acts as the backbone, while a single site coupled to the second site of the backbone serves as pendant group. Figure $4 \mathrm{~b}$ shows the calculated transmission coefficient $T(E)$ as a function of energy $E$. A Fano-resonance appears at an energy of about $0.5 \mathrm{eV}$, which is associated with the site energy of the bound state. The various transmission maxima are Fabry-Pérot resonances of the backbone channel. To calculate the differential conductance characteristic $\mathrm{d} I / \mathrm{d} V_{\mathrm{b}}\left(V_{\mathrm{b}}, V_{\mathrm{g}}\right)$ of the device for different gate voltages $V_{\mathrm{g}}$, bias and gate voltage dependent transmission coefficients $T\left(E, V_{\mathrm{b}}, V_{\mathrm{g}}\right)$ were calculated for two different potential profiles, where i) the bias drops over the left and right contacts (Figure 4c); or ii) the bias drops along the device channel (Figure 4d). In the case where the bias voltage drops across the contacts (see Figure 4c), the on-site energies of the pendant group and the backbone are not influenced by the applied bias voltage. As a consequence the two anti-resonance Fano lines have the same slope as the Fabry-Pérot interference lines (see Figure 4e). In contrast, when the potential drops over the channel (see Figure 4d), the slopes of the anti-resonance lines and the backbone resonances become different (see Figure 4f). As a result of the asymmetry of the junction, one of the Fano lines almost vanishes (see section S7 in the Supplementary Information for details). Comparing the calculations in Figure 4e and $\mathrm{f}$ with the experimental data in Figure 3a, we can conclude that, firstly, the investigated junctions are asymmetric and, secondly, that a considerable portion of the applied voltage has to drop across the junction. In a more realistic model, where two hexagonal lattices are connected to various scattering regions with and without pendant groups (see Figure 5), Fano resonances can be only observed in junctions with pendant groups (see section S6 and S8 for more details). Molecular-dynamics simulations and density functional theory calculations of different atomic configurations during nanogap formation[12] further show that dangling carbon atoms and 
edge disorder can lead to Fano resonance in the transmission spectra of partially burned graphene nanogaps (see section S8 in the Supplementary Information).

In summary we investigated graphene nanoconstrictions fabricated by narrowing down bowtie shaped graphene ribbons using a feedback controlled electroburning technique. In the case of weakly-coupled constrictions, the transport is dominated by Coulomb blockade with addition energies up to $800 \mathrm{meV}$. In the strongly coupled regime, we observe quasi-periodic chess board like pattern in the conductance maps which we attribute to multi-mode Fabry-Pérot interferences of delocalised states whose length scale agrees with two possible resonance conditions: reflections inside the current-annealed lowdoped part of the device or reflections between the electrical contacts and the low-doped part. In some of the devices, we observe sharp anti-resonances features with a Fano line shape inside the interference regime in agreement with our tight binding modelling. We attribute these features to interferences between the extended states and localised states inside the constriction. Such sharp antiresonances have the potential to underpin the development of low-power switches, because the transmission of the structure can be tuned by a small gate voltage. Moreover, the Mott formula predicts that a high $\operatorname{d} \ln G / \mathrm{d} V_{\mathrm{g}}$ should also result in a high Seebeck coefficient[51], making such devices promising candidates for thermoelectric energy harvesting. 


\section{References}

1 Lambert, C. J. Basic concepts of quantum interference and electron transport in singlemolecule electronics. Chemical Society Reviews 44, 875-888, (2015).

2 Finch, C., Garcia-Suarez, V. \& Lambert, C. Giant thermopower and figure of merit in singlemolecule devices. Physical review b 79, 033405, (2009).

3 Rong, Y. M. \& Warner, J. H. Wired Up: Interconnecting Two-Dimensional Materials with One-Dimensional Atomic Chains. Acs Nano 8, 11907-11912, (2014).

4 Chuvilin, A., Meyer, J. C., Algara-Siller, G. \& Kaiser, U. From graphene constrictions to single carbon chains. New J Phys 11, (2009).

5 Son, Y. W., Cohen, M. L. \& Louie, S. G. Half-metallic graphene nanoribbons. Nature 444, 347-349, (2006).

6 Pezoldt, J. et al. Top gated graphene transistors with different gate insulators. Physica Status Solidi C: Current Topics in Solid State Physics, Vol 7, No 2 7, 390-393, (2010).

7 Mol, J. A. et al. Graphene-porphyrin single-molecule transistors. Nanoscale, (2015).

8 Sadeghi, H., Sangtarash, S. \& Lambert, C. J. Electron and heat transport in porphyrin-based single-molecule transistors with electro-burnt graphene electrodes. Beilstein journal of nanotechnology 6, 1413-1420, (2015).

9 Candini, A., Klyatskaya, S., Ruben, M., Wernsdorfer, W. \& Affronte, M. Graphene Spintronic Devices with Molecular Nanomagnets. Nano Lett 11, 2634-2639, (2011).

10 Lu, Y. et al. High-On/Off-Ratio Graphene Nanoconstriction Field-Effect Transistor. Small 6, 2748-2754, (2010).

11 Darancet, P., Olevano, V. \& Mayou, D. Coherent Electronic Transport through Graphene Constrictions: Subwavelength Regime and Optical Analogy. Physical Review Letters 102, (2009).

12 Sadeghi, H. et al. Conductance enlargement in picoscale electroburnt graphene nanojunctions. Proceedings of the National Academy of Sciences of the United States of America 112, 26582663, (2015). 
13 Ihn, T. et al. Graphene single-electron transistors. Materials Today 13, 44-50, (2010).

14 Allen, M. T., Martin, J. \& Yacoby, A. Gate-defined quantum confinement in suspended bilayer graphene. Nat Commun 3, (2012).

15 Moser, J. \& Bachtold, A. Fabrication of large addition energy quantum dots in graphene. Applied Physics Letters 95, (2009).

16 Ki, D. K. \& Morpurgo, A. F. Crossover from Coulomb Blockade to Quantum Hall Effect in Suspended Graphene Nanoribbons. Physical Review Letters 108, (2012).

17 Barreiro, A., van der Zant, H. S. J. \& Vandersypen, L. M. K. Quantum Dots at Room Temperature Carved out from Few-Layer Graphene. Nano Lett 12, 6096-6100, (2012).

18 Lau, C. S., Mol, J. A., Warner, J. H. \& Briggs, G. A. D. Nanoscale control of graphene electrodes. Physical Chemistry Chemical Physics 16, 20398-20401, (2014).

19 Wu, Y. M. A. et al. Large Single Crystals of Graphene on Melted Copper Using Chemical Vapor Deposition. Acs Nano 6, 5010-5017, (2012).

20 Sun, Z. Z. et al. Growth of graphene from solid carbon sources. Nature 468, 549-552, (2010).

21 Ferrari, A. C. \& Basko, D. M. Raman spectroscopy as a versatile tool for studying the properties of graphene. Nat Nanotechnol 8, 235-246, (2013).

22 Puczkarski, P. et al. Three-terminal graphene single-electron transistor fabricated using feedback-controlled electroburning. Applied Physics Letters 107, (2015).

23 Simmons, J. G. Generalized Formula for Electric Tunnel Effect between Similar Electrodes Separated by a Thin Insulating Film. J Appl Phys 34, 1793-1803, (1963).

24 Lau, C. S. et al. Redox-Dependent Franck-Condon Blockade and Avalanche Transport in a Graphene-Fullerene Single-Molecule Transistor. Nano Lett 16, 170-176, (2016).

25 Burzurí, E. et al. Sequential Electron Transport and Vibrational Excitations in an Organic Molecule Coupled to Few-Layer Graphene Electrodes. ACS Nano, (2016).

26 Hanson, R., Kouwenhoven, L. P., Petta, J. R., Tarucha, S. \& Vandersypen, L. M. K. Spins in few-electron quantum dots. Reviews of Modern Physics 79, 1217-1265, (2007).

27 Bischoff, D. et al. Localized charge carriers in graphene nanodevices. Applied Physics Reviews 2, 031301, (2015). 
28 Muñoz-Rojas, F., Jacob, D., Fernández-Rossier, J. \& Palacios, J. J. Coherent transport in graphene nanoconstrictions. Physical Review B 74, 195417, (2006).

29 Miao, F. et al. Phase-coherent transport in graphene quantum billiards. Science 317, 15301533, (2007).

30 Oksanen, M. et al. Single-mode and multimode Fabry-Perot interference in suspended graphene. Physical Review B 89, 121414, (2014).

31 Bischoff, D., Libisch, F., Burgdorfer, J., Ihn, T. \& Ensslin, K. Characterizing wave functions in graphene nanodevices: Electronic transport through ultrashort graphene constrictions on a boron nitride substrate. Physical Review B 90, (2014).

32 Rickhaus, P. et al. Ballistic interferences in suspended graphene. Nat Commun 4, (2013).

33 Gunlycke, D. \& White, C. T. Graphene interferometer. Applied Physics Letters 93, (2008).

34 Kretinin, A. V., Popovitz-Biro, R., Mahalu, D. \& Shtrikman, H. Multimode Fabry-Perot Conductance Oscillations in Suspended Stacking-Faults-Free InAs Nanowires. Nano Lett 10, 3439-3445, (2010).

35 Liang, W. J. et al. Fabry-Perot interference in a nanotube electron waveguide. Nature 411, $665-669,(2001)$.

36 Morozov, S. V. et al. Strong suppression of weak localization in graphene. Phys Rev Lett 97, (2006).

37 Staley, N. E., Puls, C. P. \& Liu, Y. Suppression of conductance fluctuation in weakly disordered mesoscopic graphene samples near the charge neutral point. Physical Review B 77, (2008).

38 Berger, C. et al. Electronic confinement and coherence in patterned epitaxial graphene. Science 312, 1191-1196, (2006).

39 Hwang, C. et al. Fermi velocity engineering in graphene by substrate modification. Scientific Reports 2, (2012).

40 Ki, D. K., Jeong, D., Choi, J. H., Lee, H. J. \& Park, K. S. Inelastic scattering in a monolayer graphene sheet: A weak-localization study. Physical Review B 78, (2008). 
41 Lara-Avila, S. et al. Disordered Fermi Liquid in Epitaxial Graphene from Quantum Transport Measurements. Phys Rev Lett 107, (2011).

42 Baker, A. M. R. et al. Weak localization scattering lengths in epitaxial, and CVD graphene. Physical Review B 86, (2012).

43 Ryu, S. et al. Atmospheric Oxygen Binding and Hole Doping in Deformed Graphene on a SiO2 Substrate. Nano Lett 10, 4944-4951, (2010).

44 Cheianov, V. V. \& Fal'ko, V. I. Selective transmission of Dirac electrons and ballistic magnetoresistance of n-p junctions in graphene. Physical Review B 74, 041403, (2006).

45 Qi, Z. J. et al. Electronic Transport of Recrystallized Freestanding Graphene Nanoribbons. Acs Nano 9, 3510-3520, (2015).

46 Miroshnichenko, A. E., Flach, S. \& Kivshar, Y. S. Fano resonances in nanoscale structures. Rev Mod Phys 82, 2257-2298, (2010).

47 Verduijn, J. et al. Coherent transport through a double donor system in silicon. Applied Physics Letters 96, (2010).

48 Babić, B. \& Schönenberger, C. Observation of Fano resonances in single-wall carbon nanotubes. Physical Review B 70, 195408, (2004).

49 Gores, J. et al. Fano resonances in electronic transport through a single-electron transistor. Physical Review B 62, 2188-2194, (2000).

50 Clerk, A. A., Waintal, X. \& Brouwer, P. W. Fano resonances as a probe of phase coherence in quantum dots. Physical Review Letters 86, 4636-4639, (2001).

51 Cutler, M. \& Mott, N. F. Observation of Anderson Localization in an Electron Gas. Physical Review 181, 1336-1340, (1969). 


\section{Acknowledgements}

We thank the Royal Society for a Newton International Fellowship for J. A. M., the Agency for Science Technology and Research (A*STAR) for a studentship for C.S.L. and a University Research Fellowship for J. H. W. This work is supported by Oxford Martin School, the European Commission (EC) FP7 ITN "MOLESCO” (project no. 606728) and UK EPSRC (grant nos. EP/K001507/1, $\mathrm{EP} / \mathrm{J} 014753 / 1, \mathrm{EP} / \mathrm{H} 035818 / 1$ and EP/J015067/1). This project/publication was made possible through the support of a grant from Templeton World Charity Foundation. The opinions expressed in this publication are those of the author(s) and do not necessarily reflect the views of Templeton World Charity Foundation. The authors would like to thank D. Gunlycke for his help and the useful discussions and Y. Fan and J. Nägele for providing supporting transport data.

\section{Captions}

Figure 1. (a) False colour SEM image of a graphene constriction (grey) contacted by gold contacts (yellow). (b) Schematic of a graphene nanoconstriction device. (c) Conductance as a function of back gate voltage recorded at $V_{\mathrm{b}}=100 \mathrm{mV}$ of an as-prepared device. (d) Raman spectrum of the centre region of the graphene bow-tie after electroburing. (e) $I-V_{\mathrm{b}}$ traces recorded during feedbackcontrolled electroburning. The first and last traces are shown in blue and red, respectively.

Figure 2. Nanostructures with different electronic behaviour formed during electroburning. (a) $I-V_{\mathrm{b}}$ trace and (b) current map of an empty gap. (c) $I-V_{\mathrm{b}}$ trace and (d) current map of a weakly coupled constriction showing sequential tunnelling. (e) $I-V_{\mathrm{b}}$ trace and (f) conductance map of a strongly coupled constriction showing resonance effects. All data was recorded at $T=4 \mathrm{~K}$ under vacuum. The insets depict a scheme of the constriction.

Figure 3. (a) Conductance map at $T=4 \mathrm{~K}$ of a strongly coupled constriction showing interference effects. A sharp anti-resonance feature around $V_{\mathrm{g}}=-18 \mathrm{~V}$ can be observed. The dotted line is used to extract the lever arm. (b) Gate traces for different bias voltages $0.1 \mathrm{mV} \leq V_{\mathrm{b}} \leq 8 \mathrm{mV}$ in $0.2 \mathrm{mV}$ steps of the data shown in (a). The curves are offset by $0.2 \times 10^{3} e^{2} / h$ for clarity. (c) Gate traces at different 
bias voltages (dotted lines) and fits using Equation 1 (solid lines). The inset shows the dependence of the Fano factor $|q|$ as a function of the applied bias voltage.

Figure 4. (a) Tight-binding model of a pendant state interacting with an extended 'backbone' state. The backbone is described by a chain of 5 sites with on-site energies $\varepsilon_{1-5}$ that are coupled by hopping matrix elements $-\gamma_{1-4}$ and coupled to the leads via the outer most sites by hopping matrix elements $-\alpha_{\mathrm{L}}$ (on the left side) and $-\beta_{\mathrm{R}}$ (on the right side). The pendant group with an on-site energy $\varepsilon_{\mathrm{s}}$ is coupled to the second site of the backbone by a hopping matrix element $-\alpha$. (b) Calculated transmission coefficient as a function of energy. (c), (d) Sketch of the potential profile where (c) the bias drops over the left and right contacts and (d) the bias voltage drops along the device channel. (e), (f) Corresponding conductance maps as a function of bias and gate voltage for the cases depicted in (c), (d), respectively.

Figure 5. Transmission through graphene junctions. (a) Clean graphene ribbon connected to two graphene electrodes, (b-e) graphene junctions with different shape and position of pendent groups. The dotted circles indicate the position of Fano features. 

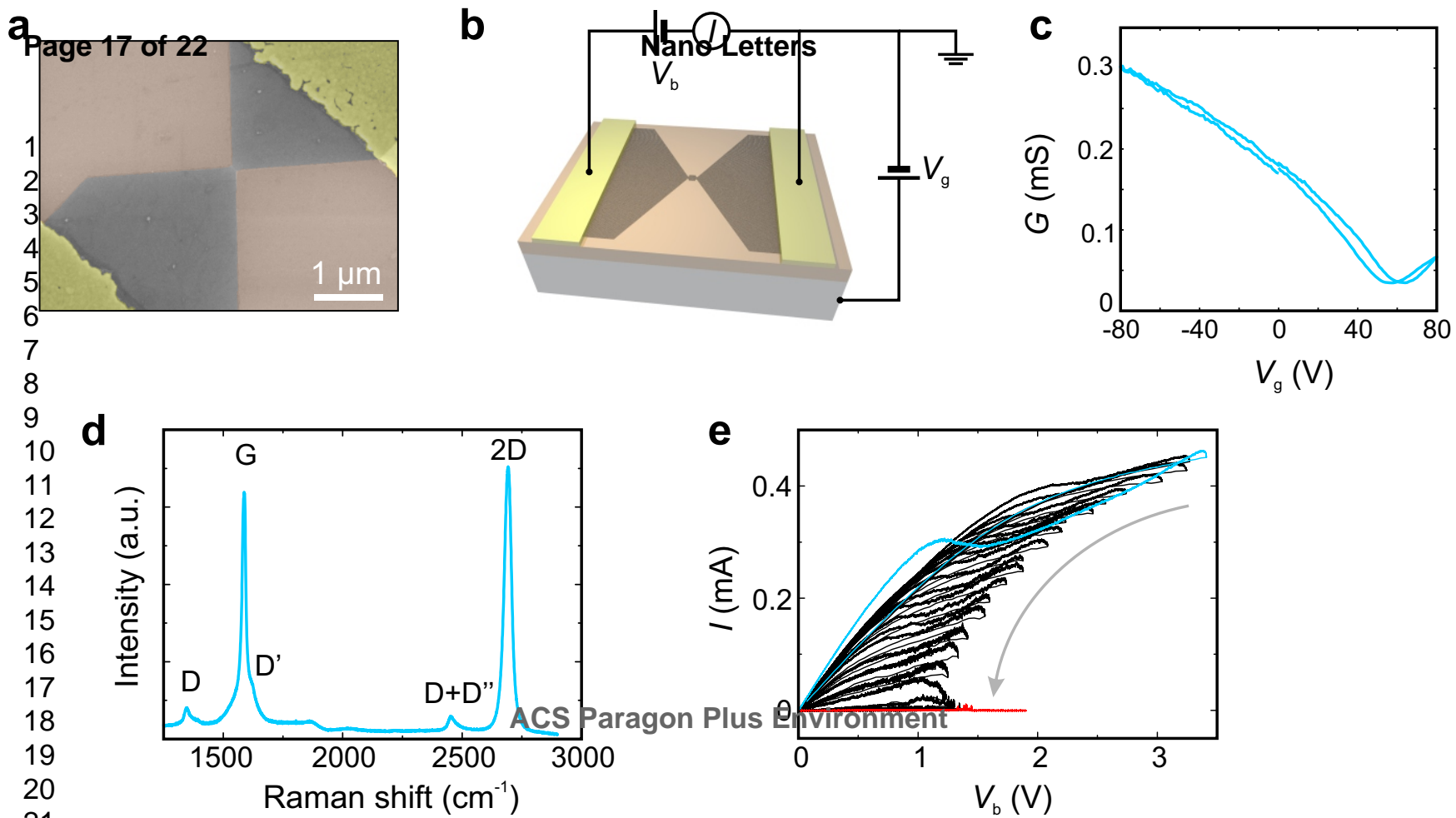


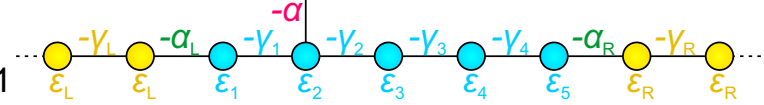

2
$b_{4}^{3}$

5

$6^{\text {- }}$

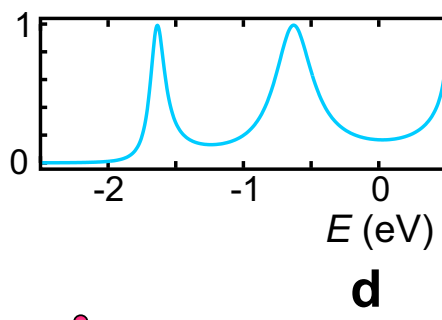

10

$\mathbf{G}_{1}$

$\frac{12}{13} 0-10$ o 0

Leąd 1 Channel Lead 2

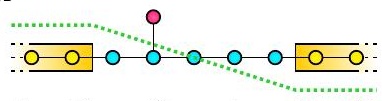

Lead 1 Channel Lead 2

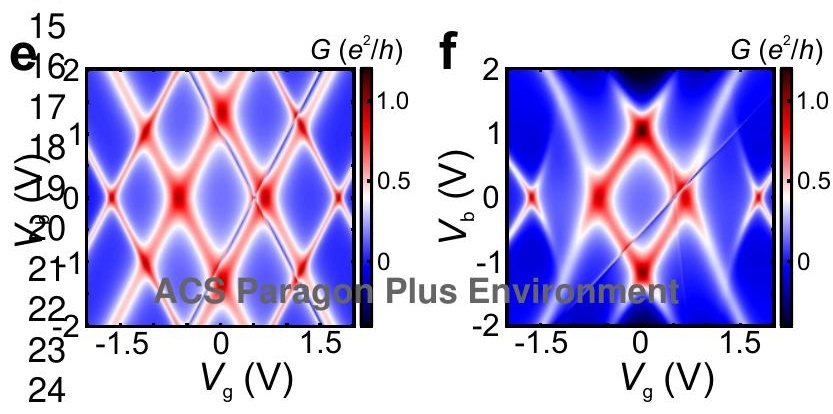




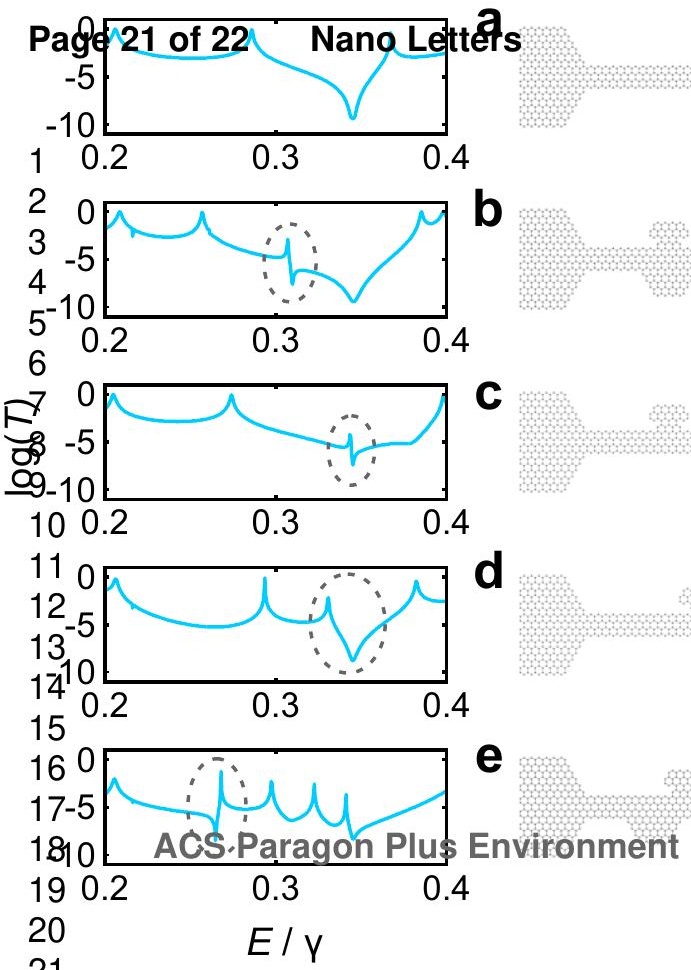




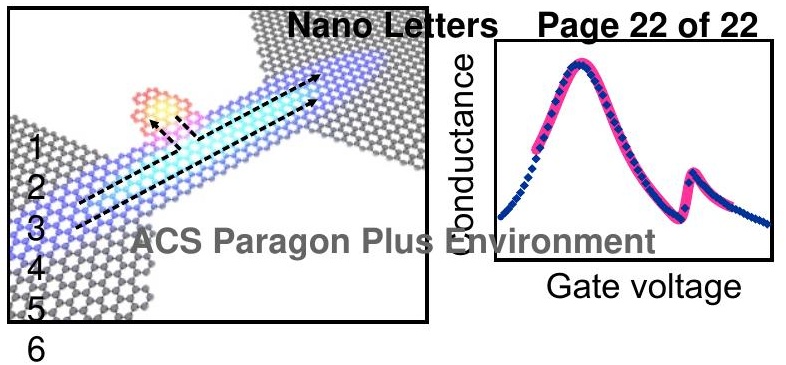

